\title{
THE POPULATION OF TWIN RELATED BOUNDARIES IN HIGH PURITY NICKEL AS MEASURED WITH NEAR-FIELD HIGH ENERGY X-RAY DIFFRACTION MICROSCOPY
}

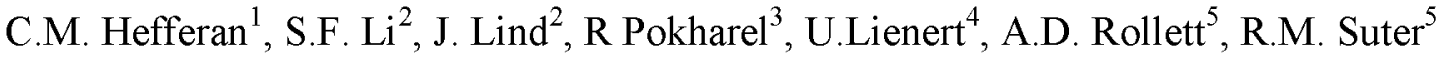 \\ ${ }^{1}$ RJ Lee Group, Inc., 350 Hochberg Rd., Monroeville, PA, 15146, USA \\ ${ }^{2}$ Lawrence Livermore National Laboratory, 7000 East Ave., Livermore, CA, 94550, USA \\ ${ }^{3}$ Los Alamos National Laboratory, Los Alamos, NM, 87545, USA \\ ${ }^{4}$ Deutsch Elektronen Synchrotron DESY, Notkestrasse 85, Hamburg, 22607, Germany \\ ${ }^{5}$ Carnegie Mellon University, 5000 Forbes Ave., Pittsburgh, PA 15213, USA
}

Keywords: twin boundary, high energy $\mathrm{X}$-ray diffraction microscopy, forward modeling orientation reconstruction, Brandon criterion

\begin{abstract}
The ability of near-field High Energy X-ray Diffraction Microscopy (nf-HEDM) to map the dynamic response of microstructures under the influence of thermo-mechanical loads has been repeatedly demonstrated. Here, we use nf-HEDM to monitor the evolution of the same high purity, polycrystalline nickel microstructure through a sequence of five, grain growth anneal states. While the microstructure coarsens in response to the applied heat, the boundary misorientation distribution was found to be both static and consistent with that of a twinned microstructure. Analysis of the $\Sigma 3^{\mathrm{n}}$ grain boundaries shows that in addition to accounting for a large proportion of the grain boundary area, these twin related boundaries also exhibit minimal deviation from the ideal $\Sigma 3^{\mathrm{n}}$ configuration.
\end{abstract}

\section{Introduction}

The scientific instrumentation made available to the materials community in recent decades has expanded our understanding of both how materials are assembled and what governs their behavior while in service. Orientation imaging microscopy (OIM) [1,2] is one field that has seen dramatic advances, which has led to new ways of interpreting microstructural data. Historically, the determination of texture in bulk materials was primarily confined to the generation of X-ray pole figures. Now, with techniques such as electron backscatter diffraction microscopy (EBSD) [3] and synchrotron-based techniques [4-8] like near-field High Energy X-ray Diffraction Microscopy (nf-HEDM) [9], correlating grain orientations with the spatial locations within a polycrystal has become routine. With the ability to non-destructively measure bulk microstructures, nf-HEDM is well-suited for direct, one-to-one measurements of the polycrystalline response to thermo-mechanical processes [10-13].

Near-field High Energy X-ray Diffraction Microscopy is an orientation imaging technique, based on synchrotron generated X-rays, for non-destructive measurement of diffraction from a polycrystalline sample. The experimental geometry of the technique is illustrated in Figure 1a. 
This is a simplified summary of the apparatus currently used at Advanced Photon Source 1-ID beamline at Argonne National Laboratory [8,9]. High energy ( $>50 \mathrm{keV}) \mathrm{X}$-rays are microfocused and imparted upon a polycrystalline sample, which produces Bragg scattering when a given grain satisfies the diffraction condition. To permit multiple reflections for each grain within the microstructure, the sample is rotated while imaged over fixed intervals. Imaging of this diffraction is performed in the near-field regime, with sample-to-camera distances of only a few millimeters.

By using both high resolution cameras ( $\sim 1 \mu \mathrm{m}$ pixels) and multiple camera-to-sample distances, we can achieve precise spatial resolution of the crystallographic orientations within the sample. The mapping can be extended to the third dimension through the vertical translation of the sample, such that the micro-focused beam illuminates a new planar section. This mapping is analogous to serial sectioning, with the added benefit that imaging is not restricted to the sample surface.

Figure $1 \mathrm{~b}$ conceptually demonstrates the forward modeling method (FMM) [14] grain mapping procedure, which is a computer simulation that replicates the synchrotron experiment. Sample space is tessellated with a fine grid, which is assigned crystallographic orientations through a search in orientation space. By optimizing the overlap between the experimental diffraction images and the simulated diffraction signals produced by these grid assignments, orientation maps are generated with high spatial and orientation resolution. An example map from the nickel microstructure measured in this study is shown in Figure 1c, where unique orientations have been assigned colors based on their Rodrigues-Frank orientation representation [15].

In this paper, we use nf-HEDM to investigate the grain boundary distribution of a high purity (99.999\%) nickel microstructure after a sequence of grain growth anneals. Due to their contribution in governing macroscopic properties such as susceptibility [16], conductivity [17], toughness [18] and corrosion resistance [19], gaining a fundamental understanding of the evolution of grain boundaries under external forces is informative. This coarsening experiment consisted of mapping the same volume of material after $800^{\circ} \mathrm{C}, \sim 30$ minute anneals, so that differential changes in the microstructure can be tracked and quantified.

This paper focuses on the properties of the microstructure's grain boundary misorientation distribution, which is one of the fundamental metrics used to describe the grain boundary network. While remaining approximately static during the annealing sequence, this misorientation distribution does exhibit exceptional information in the context of special, twin boundaries, commonly found in low stacking fault energy materials. We interpret these boundaries with the aid of coincident site lattice (CSL) theory [20], which is commonly used to describe the geometry of misoriented lattices. Special consideration is given to the significant population associated with the twin distribution in the microstructure.

\section{Experiment}

A $1 \mathrm{~mm}$ diameter, high purity $(99.999 \%)$ nickel cylinder was prepared for a grain coarsening measurement [21]. The initial microstructure was fully recrystallized and was composed of 
grains with an average volume of $10^{4} \mu^{3}$ and containing approximately 2,000 grains. No appreciable orientation texture was present in the initial sample state.

The grain coarsening measurement was performed at the Advanced Photon Source 1-ID beam line, using nf-HEDM. The sample was annealed ex-situ in forming gas at $800^{\circ} \mathrm{C}$ for intervals of 23-35 minutes. After each incremental anneal, the same volume of microstructure was measured with the near-field HEDM procedure and reconstructed using the forward modeling method. A total of six sample states were measured.

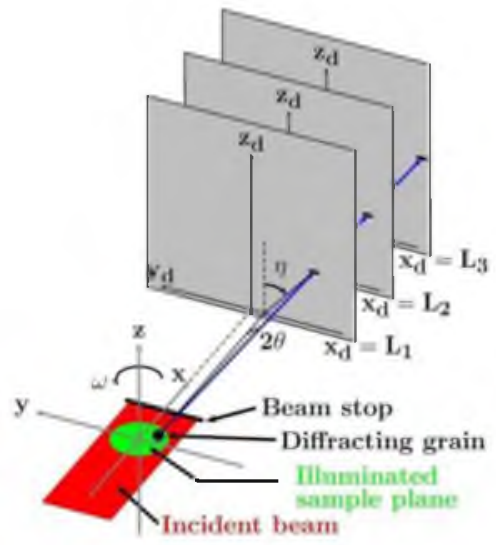

(a)

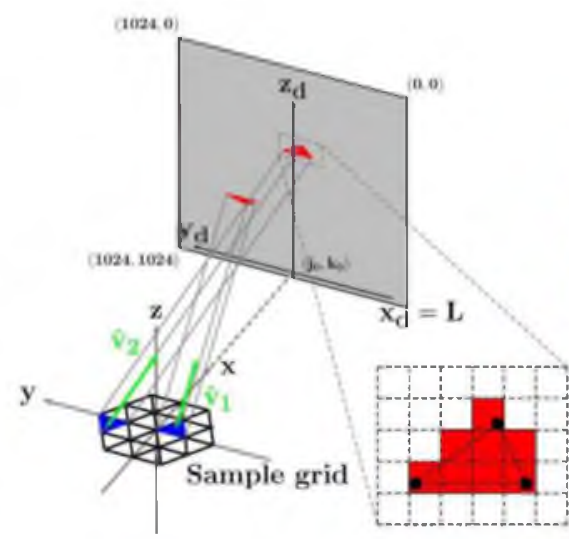

(b)

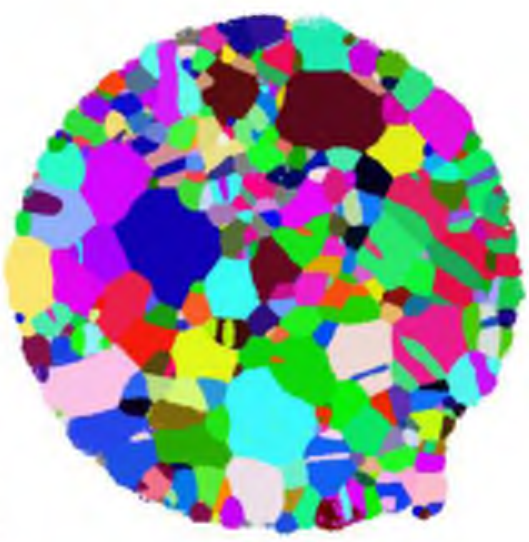

(c)

Figure 1. Illustration of (a) the setup used at the Advanced Photon Source 1-ID beamline for nf-HEDM. A planar, micro-focused x-ray beam (red) is incident on a polycrystalline sample (green). In this configuration, a grain (black) satisfies the Bragg diffraction condition and produces a diffraction peak that can be imaged on high resolution cameras, situated a few millimeters downstream of the sample. The forward modeling method (FMM) is conceptually illustrated in (b), where the sample location has been replaced by a triangular grid. Two triangles have been assigned a crystallographic orientation (blue), which results in Bragg peaks in the simulated detector. The a representative nickel microstructure map, reconstructed using nf-HEDM with FMM is shown in (c). Each nickel sample state contains between 70 to 100 such maps. Crystallographic orientations have been converted to the Rodrigues-Frank representation and different orientations show up as distinct colors.

The diffraction experiment was performed with an operating X-ray energy of $64.351 \mathrm{keV}$ and used two rotation axis-to-detector distances $(4.8,6.8 \mathrm{~mm})$. Intermittently, a third distance of 8.8 $\mathrm{mm}$ was employed for reconstruction calibration. The focused beam had a FWHM of $4 \mu \mathrm{m}$. The diffraction images were collected on a $2048 \times 2048$ CCD camera, with effective, square pixel sizes of $1.48 \mu \mathrm{m}$, utilizing a free standing, single crystal, LuAG scintillator. Given the experimental geometry of this near-field HEDM setup, each grain produced between 49 and 64 distinct diffraction peaks. 
The collected volumes of microstructure were composed of circular, planar sections in $4 \mu \mathrm{m}$ spacings, with the initial volume spanning $280 \mu \mathrm{m}$ length. To accommodate growth in average grain size with each anneal, the size of the volume was expanded to include more planar sections after each successive heat treatment.

Each layer of microstructure was reconstructed using the forward modeling method. The sample was reconstructed on an equilateral triangular grid composed of $2.81 \mu \mathrm{m}$ sides, with each triangle assigned an optimal crystallographic orientation. Layers were stacked and converted into a $3 \mathrm{D}$ rectilinear grid, with voxels optimally chosen [21] to be $1.2 \mu \mathrm{m} \times 1.2 \mu \mathrm{m} \times 4 \mu \mathrm{m}$. Each voxel was assigned a grain number, which is associated with an average orientation, based upon the averaging of all voxels assigned to the grain. Given the minimal mosaicity of the microstructure, a $3^{\circ}$ misorientation threshold was used to define grain boundaries in the mesh.

The rectilinearly tessellated grid was smoothed into a three dimensional microstructure using software built upon the Computational Geometry Algorithms Library (CGAL) [22]. A Delaunay triangulation routine was used to produce a surface mesh, capturing the grain boundary network $[23,24]$, while a volumetric mesh was generated using tetrahedral elements. Using these routines, the average initial state grain consisted of $\sim 1200$ reconstructed triangles. Each triangle's optimal orientation varied from the grain averaged orientation by $<0.1^{\circ}$, which is resolvable with the technique $[10,11]$.

\section{Results}

The measured misorientation angle distribution of the nickel microstructure is shown in Figure 2, where the misorientation angle has been partitioned with $0.25^{\circ}$ bins. With all anneal states exhibiting approximately the same misorientation angle distribution [25], only the initial and final (Anneal5) states have been displayed. The distribution has been weighted by both interface area and number fraction, with the latter used to eliminate biasing due to large area boundaries.

From Figure 2, it is evident that a large population of boundaries falls into a small collection of misorientation bins. Specifically, the $60^{\circ}$ bin accounts for approximately $14 \%$ of all boundaries in the measured volume and $>25 \%$ of the total interfacial area. The boundary fraction plot also shows that an appreciable population of boundaries exists between $30^{\circ}$ and $40^{\circ}$. Outside of these select few bins, the distribution is consistent with that of a random polycrystal, as described by the empirical Mackenzie distribution [26]. These histograms are the result of binning 19,510 unique boundaries for the initial state and 13,957 boundaries for the final state.

The distributions shown in Figure 2 indicate a microstructure containing many twin boundaries [27]. Geometrically, twin boundaries exist as $60^{\circ}<111>$ rotations in face-centered cubic materials and are frequently observed in thermally processed, low stacking fault energy materials. In addition to their unique geometry, coherent twins have many special properties, including low mobility and energy $[28,29]$ when the boundary plane is $\{111\}$.

While the twin misorientation can directly explain the large $60 \pm 0.125^{\circ}$ bin population in Figure 2 , the three smaller populations between $30^{\circ}$ and $40^{\circ}$ can also be attributed to twin boundaries.

With grain boundaries terminating at triple lines and the microstructure possessing many twin boundaries, it is highly probable that two different twin boundaries will terminate at a triple line. 
During such occurrences, the misorientation of the third boundary is determined and associated with a $38.94^{\circ}<110>$ misorientation, commonly known as a $\Sigma 9$ in the language of CSLs. Further, when a twin $(\Sigma 3)$ and $\Sigma 9$ meet at a triple line a $\Sigma 27$ is formed. Consequently, a microstructure containing many twins should have many $\Sigma 3^{\mathrm{n}}$ boundary misorientations [30]. These are the origins of the additional peaks in Figure $2 \mathrm{c}$, where the three peaks are associated with $\Sigma 9, \Sigma 27 \mathrm{a}$, and $\Sigma 27 \mathrm{~b}$ have misorientation angles of $38.94^{\circ}, 31.56^{\circ}$, and $35.43^{\circ}$, respectively.

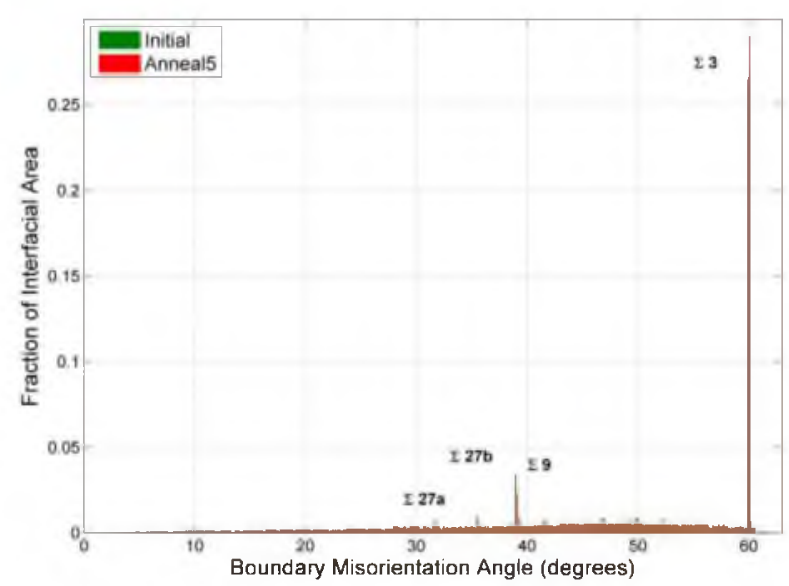

(a)

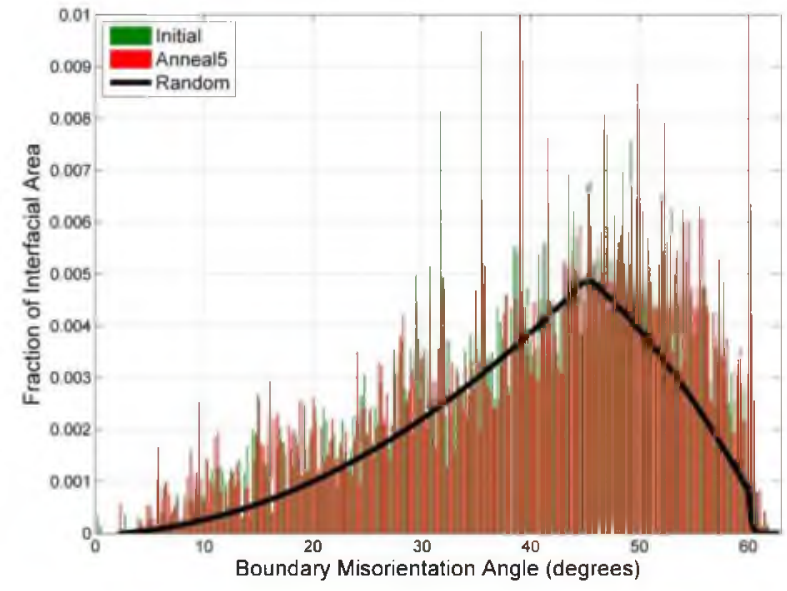

(b)

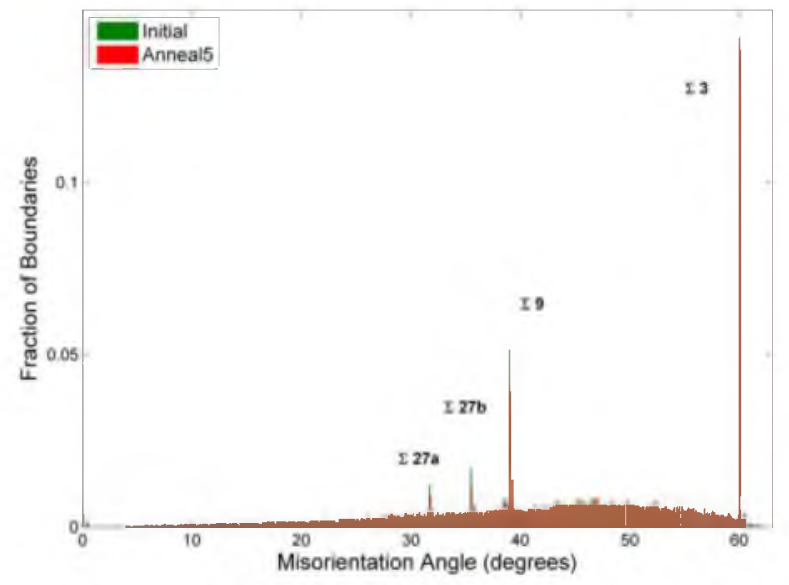

(c)

Figure 2. Misorientation distribution of high purity nickel microstructure in the initial, recrystallized state (green) and after five anneals of $\sim 30$ minutes at $800^{\circ} \mathrm{C}$ (red). The distribution has been generated with $0.25^{\circ}$ bins and has been (a) area weighted and (c) number weighted. A scaled version of the Mackenzie distribution [26] has also been outlined in black on the area weighted histogram (b) to demonstrate the underlying random distribution of boundary misorientations. While the measured regions of microstructure had significant overlap, a grain-to-grain correlation has not yet been performed between the initial and Anneal5 states, so variations are possible between the displayed states. Specifically, 19,510 unique boundaries are present in the initial microstructure, while 13,957 boundaries are in Anneal5. Special boundaries have been labeled with their CSL designation. 
While appreciably populated, these twin related misorientations also exhibit a significant sharpness in the misorientation distribution, with each $\Sigma 3^{n}$ appearing to reside in a single $0.25^{\circ}$ bin. Recalling that the misorientation distribution is approximately static, we produce the area weighted misorientation angle distribution with $0.01^{\circ}$ bin resolution for the initial state microstructure. This finely binned distribution is shown in Figure 3 for the $\Sigma 3$ and $\Sigma 9$ misorientation peaks.

The sharpness of the misorientation peaks is markedly illustrated, with nearly $10 \%$ of the entire interfacial area of the measured polycrystal having a misorientation angle within $0.005^{\circ}$ of the twin misorientation angle. Such sharpness has been observed with previous nf-HEDM measurements [31], but never with such robust statistics. The asymmetry of the $\Sigma 3$ peak can attributed to the three-fold symmetry of the $\langle 111\rangle$ axis in cubic crystals. Specifically, misorientations in excess of $60^{\circ}$ are symmetry reduced to below $60^{\circ}$ in cubic misorientation space.

Given the sharpness of the misorientation angle distribution near the $\Sigma 3^{\mathrm{n}}$ misorientation configurations, we expand from the scalar representation of misorientation and quantify misorientation space using the full three parameter misorientation, which includes misorientation axis in addition to rotation angle. To perform a systematic evaluation of the distribution of boundaries in misorientation space, we use a collection of points in CSL space as explicitly defined misorientations. Then for each grain boundary in the nickel microstructure, we can

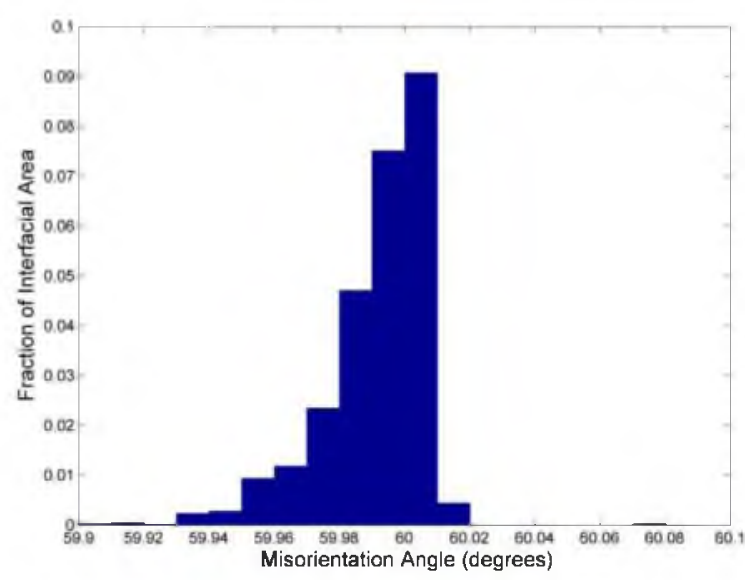

(a)

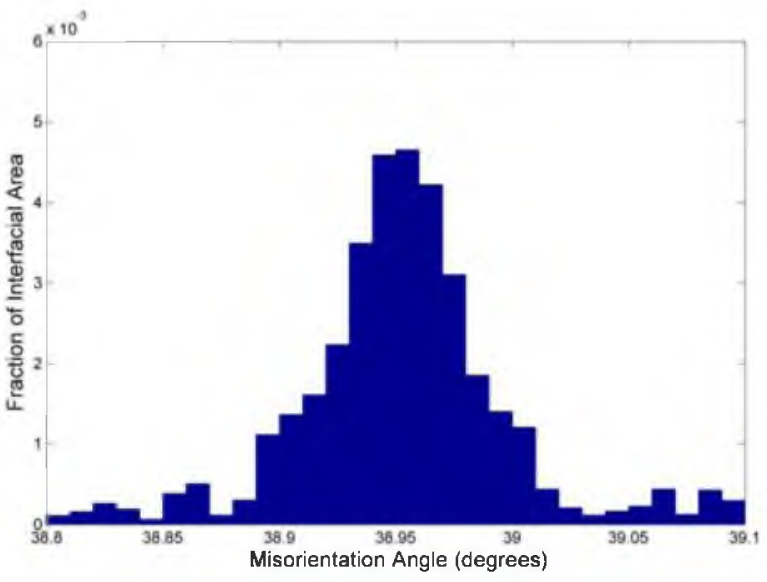

(b)

Figure 3. Misorientation angle distribution centered at (a) $60^{\circ}$ and (b) $38.94^{\circ}$, using $0.01^{\circ}$ bins. The initial state grain boundary distribution has been used for both plots. The peak on the left indicates that $\sim 10 \%$ of the interfacial area has a misorientation angle within $\pm 0.005^{\circ}$ of $60^{\circ}$. The asymmetry of the $60^{\circ}$ peak can be attributed to the three-fold symmetry on $\langle 111\rangle$.

compute a distance to each point in the defined CSL space. For this operation, we define our distance, $\Delta \mathrm{g}$, from a CSL as

$$
\Delta \mathrm{g}=\left(\Delta \mathrm{g}_{\Sigma}\right)^{-1} \Delta \mathrm{g}_{\text {boundary }}
$$


where $\Delta \mathrm{g}_{\text {boundary }}$ is the misorientation of the measured grain boundary and $\Delta \mathrm{g}_{\Sigma}$ is the CSL configuration. In effect, $\Delta \mathrm{g}$ measures a misorientation between misorientations. Consequently, $\Delta \mathrm{g}$ can be handled like a misorientation.

Using 97 distinct CSL values, ranging from $\Sigma 3$ to $\Sigma 133$ [20], each boundary has been assigned a distance from these 97 configurations, which is the rotation angle associated with $\Delta \mathrm{g}$. For a given boundary, the CSL with the minimum distance is assigned as the closest CSL point to that grain boundary. Using these associations, we are able to analyze the area weighted population of grain boundaries as a function of closest CSL point.

The CSLs that have significant populations associated with them are illustrated in Figure 4, where two methods of classification are displayed. The first set of data, shown as red points, takes all boundaries that are within $0.25^{\circ}$ of its nearest CSL and defines that as a distribution. Consequently, not all boundaries in the microstructure are included in this population. The total area of this subset of boundaries is then tabulated and the area fraction that is associated with each CSL is listed. Thus, the red dot near unity for $\Sigma 3$ indicates that nearly all boundaries that are within $0.25^{\circ}$ of a CSL are within $0.25^{\circ}$ of the $\Sigma 3$.

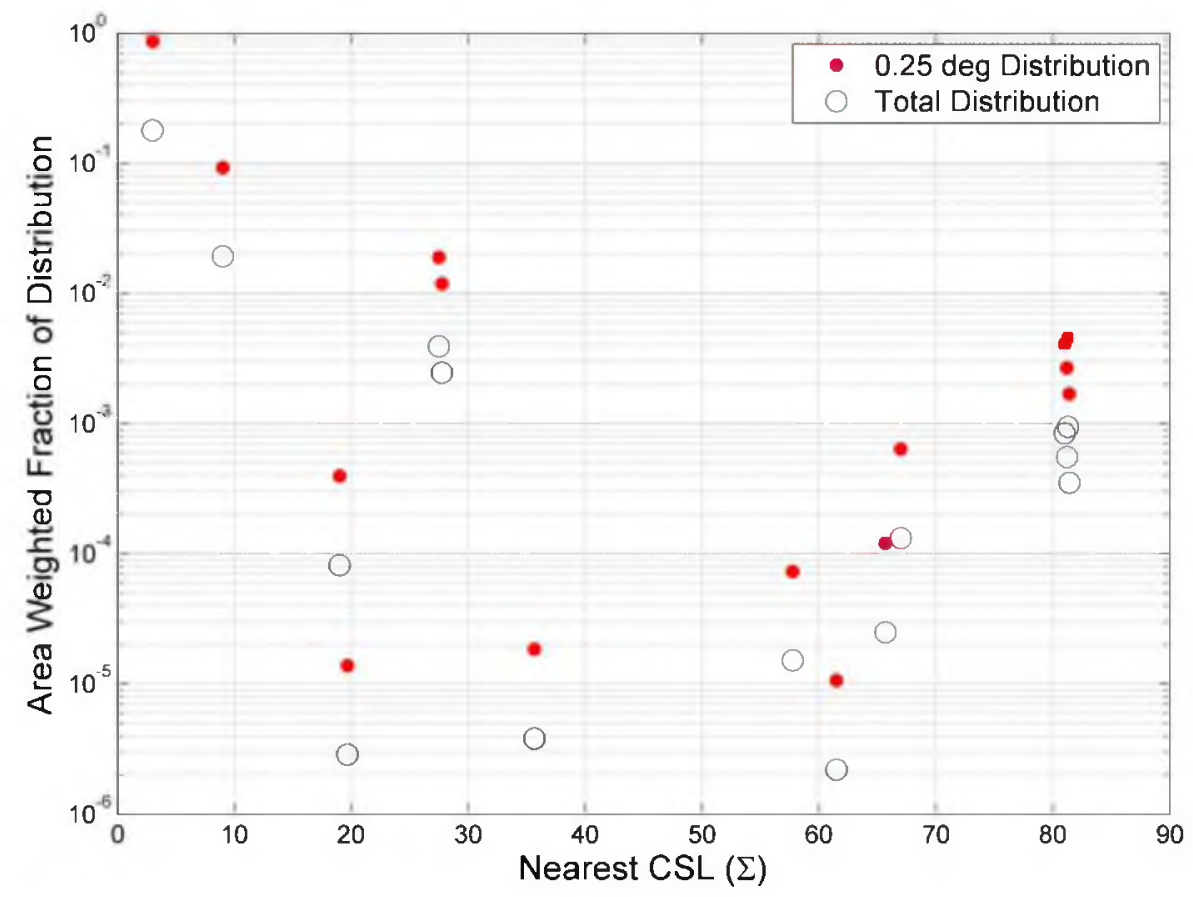

Figure 4. Area fraction of microstructure associated with nearest coincident site lattice point $(\Sigma)$. Red dots show the distribution of boundaries that are within $0.25^{\circ}$ of a CSL, while circles in black display the area fraction of all boundaries by nearest CSL association. Points with the same CSL value, but different geometrical descriptions ( $\Sigma 27 \mathrm{a}, \Sigma 27 \mathrm{~b})$ have been offset for clarity.

The black circle population in Figure 4 relaxes this $0.25^{\circ}$ criterion and measures the area fraction of boundaries associated with a given CSL. Thus, all boundaries in the microstructure are 
included in this population. Using this partitioning, $\sim 15 \%$ of the interfacial area in the microstructure is closer to the $\Sigma 3$ than the 96 other CSLs.

Figure 4 illustrates the nearness to an exact CSL configuration for a given collection of grain boundaries. Of specific note is that the eight CSLs with the largest area fraction in the $0.25^{\circ}$ distribution can all be described as $\Sigma 3^{\mathrm{n}}$. While noting that these boundaries appear very close to the exact $\Sigma 3^{\mathrm{n}}$ configuration, the choice of $0.25^{\circ}$ for partitioning the data is arbitrary. We could use the Brandon criterion [32] of $15^{\circ} \Sigma^{-1 / 2}$ as the threshold radius, but given that the Brandon criterion is larger than the $0.25^{\circ}$ partition already used $\left(\sim 8.66^{\circ}\right.$ for $\left.\Sigma 3\right)$, it appears we would be making our partition coarser than the experimental signal. We propose to investigate the behavior of this threshold angle for the $\Sigma 3, \Sigma 9, \Sigma 27 \mathrm{a}$, and $\Sigma 27 \mathrm{~b}$.

The evaluation of cutoff angle for CSL association is demonstrated in Figure 4. Here, we take the data associated with a black circle from Figure 4, which is a collection of all boundaries that are closest to a given CSL, and evaluate the cumulative area distribution as a function of distance from the CSL configuration. All six sample states have been plotted for completeness.

It is notable that all distributions exhibit the same error function shape. This is also true of the four $\Sigma 81$ boundaries, which are not shown. In increasing the CSL threshold, boundaries are added up to a given distance (the top of the error function), at which point increasing the threshold does not significantly add grain boundaries to the $\Sigma 3^{\mathrm{n}}$ population. Past this "plateau" region, a second tail is present and can be interpreted as the inclusion of random boundaries to the given CSL. If the density of CSL was made larger, presumably these random boundaries would become associated with a different point in CSL space.

For comparison, the Brandon criterion angle has been plotted as a vertical line on all four graphs. Achieving their purpose, these Brandon lines tend to demarcate the random boundary cutoff for the $\Sigma 27$ boundaries, with a slight overestimation. For the $\Sigma 9$ distribution, the transition occurs at a much lower threshold angle than the Brandon criterion. Due to the density of the CSL points, the $\Sigma 3$ does not demonstrate the random boundary tail of the other three graphs. It would be informative to decrease the density of CSL space used and determine where the random boundary transition occurs for the $\Sigma 3$. This has been identified as future work. Averaging each distribution over sample states, and fitting to an error function, yields a distribution center that is $0.29 \pm 0.02^{\circ}$ and constant in $\Sigma$.

\section{Discussion}

Although dynamic data was available, we focused on a metric that remained approximately static in response to annealing. With such a complicated data set, consisting of thousands of grains and tens of thousands of boundaries, identifying the slowly varying or static quantities assists in providing a benchmark for comparison with the dynamic variables in the system. Moving forward, it is obvious that looking at quantities such as grain boundary normals and grain size will be most informative, especially when correlated between measurement states.

The misorientation distribution in Figure 2 displayed both the initial and final states of the 
measurement, and while appearing approximately static, the microstructure does exhibit some nuanced differences including population shifts in the $\Sigma 3$ and $\Sigma 9$ bins. Specifically, the area population of $\Sigma 3$ boundaries appears to have increased with annealing, while the $\Sigma 9$ bin has been depopulated. The $\Sigma 3$ increase is shown to be cumulative when compared with the other four

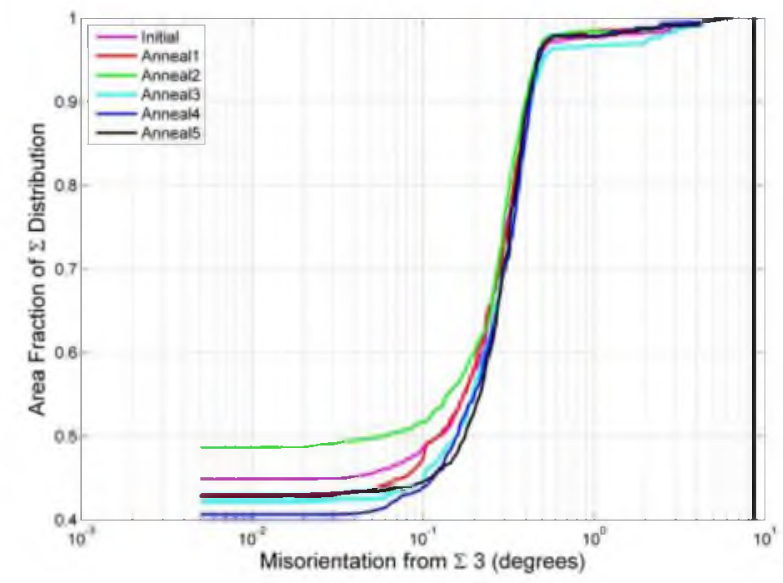

(a) $\Sigma 3$

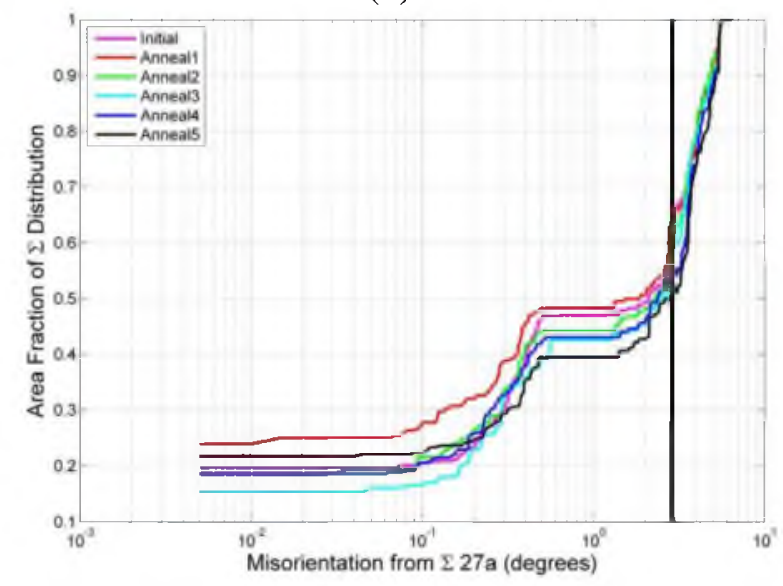

(c) $\Sigma 27 \mathrm{a}$

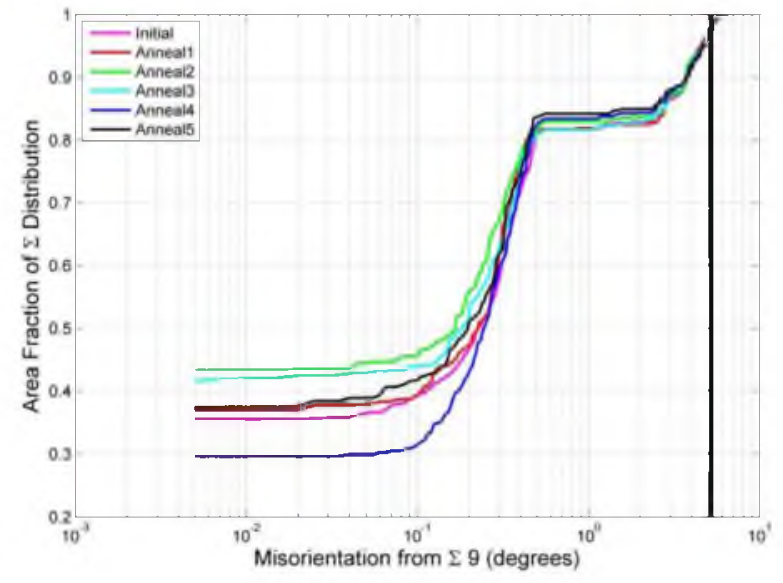

(b) $\Sigma 9$

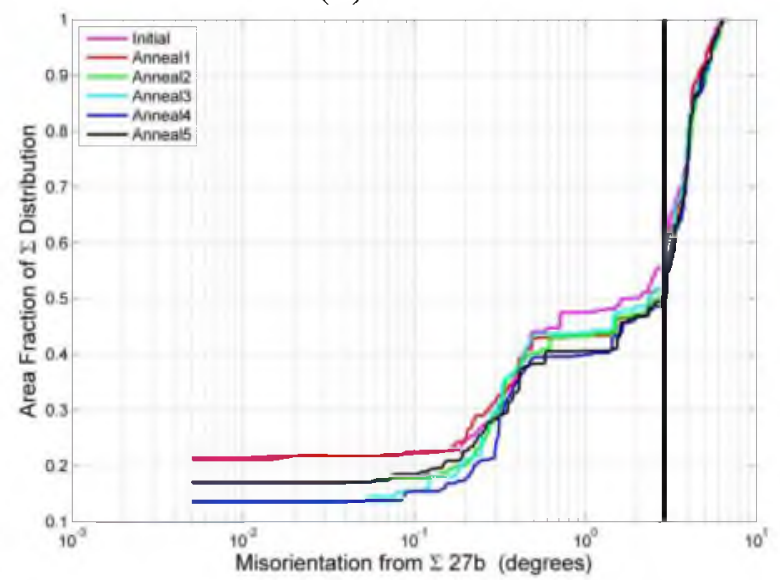

(d) $\Sigma 27 b$

Figure 5. Integrated area distributions as a function of distance from (a) $\Sigma 3$, (b) $\Sigma 9$, (c) $\Sigma 27$ a, and (d) $\Sigma 27 b$ configuration. The vertical axis indicates the fractional area of boundaries (associated with a given CSL) that are within the misorientation from the exact CSL indicated on the horizontal axis. The vertical black line indicates the Brandon criterion [31] for the given CSL.

sample states. A correlation between grains among sample states should provide information as to whether a static grain boundary distribution is present, and if these changes are due to growth of $\Sigma 3 \mathrm{~s}$ at the expense of $\Sigma 9 \mathrm{~s}$. Answering such questions in the context of boundary mobility and energy would provide a complete picture of the interface network's response to a key driving force for microstructural change. Further, the inclusion of grain boundary plane information to 
misorientation will allow the grain boundary character distribution (GBCD) [33] to be fully described. Monitoring the evolution of tracked $\Sigma 3^{\mathrm{n}}$ boundaries should be most informative.

In addition to subtle changes in the population of boundary types, the sharpness of the misorientation distribution at twin related boundaries $\left(\Sigma 3^{\mathrm{n}}\right)$ demonstrates a unique physical signal within the microstructure. Figure 4 shows a large population of $\Sigma 3^{n}$ boundaries was found to be exceedingly close to the exact CSL configuration. When explored as distance from the CSL (Figure 5), an intrinsic signal for configuration clustering was observed. Boundaries near the $\Sigma 3^{\mathrm{n}}$ were found to be nearly coincident with the CSL configuration and relaxing the distance requirement for association with the $\Sigma 3^{\mathrm{n}}$ configuration lead to a region where few additional boundaries were identified. The mean distance from the exact $\Sigma 3^{\mathrm{n}}$ for all four boundaries was shown to be independent of $\Sigma$, while the entire population could be captured with a distance of approximately $0.5^{\circ}$.

It is quite apparent that this six state annealing experiment is a statistically robust data set. The ability to see one-to-one changes in both grains and grain boundaries as coarsening occurs should be of great benefit. While undoubtedly of value to the materials community, the results of these measurements can also be viewed in the realm of data mining. With thousands of grains and tens of thousands of boundaries needing to be tracked through multiple sample states, the computational challenges are substantial. Further, tracking critical events, such as boundary extinction or grain nucleation will require advanced software that is currently in development.

Finally, the relevance of this work to processing and fatigue in superalloys should be emphasized. The capability demonstrated here will allow detailed and statistically significant volumes of more complex materials to be studied as they undergo thermal and/or mechanical processing. It has been shown that the nf-HEDM technique is able to map orientations even in deformed materials $[11-13,34]$. Further, successful reconstructions have been performed on intermetallic microstructures, where crack initiation was investigated [34-36]. Such studies will allow detailed testing and validation of computational models that, with such testing, can form a basis for more rapid development of new materials.

\section{Acknowledgements}

This work was supported primarily by the Metals and Nanostructures program of the National Science Foundation under Award Numbers DMR-0805100 and DMR-1105173. Part of this work was performed under the auspices of the U. S. Department of Energy by Lawrence Livermore National Laboratory under Contract DE-AC52-07NA27344. The use of high performance computing facility was supported in part by the National Science Foundation through TeraGrid resources provided by Texas Advanced Computing Center under Grant No. DMR080072. Use of the Advanced Photon Source was supported by the US Department of Energy, Office of Science, Office of Basic Energy Sciences, under Contract No. DE-AC02$06 \mathrm{CH} 11357$. 


\section{References}

1. B.L. Adams, S.I. Wright, and K. Kunze, "Orientation imaging: the emergence of a new microscopy," Metallurgical Transactions A, 24(4) (1993), 819-831.

2. D.P. Field, "Recent advances in the application of orientation imaging," Ultramicroscopy, 67(1) (1997), 1-9.

3. M. Kumar, B.L. Adams, and D.P. Field, Electron backscatter diffraction in materials science (New York, NY: Springer, 2009).

4. H. Poulsen, Three-dimensional x-ray diffraction microscopy (Berlin, Germany: Springer, 2004).

5. B. Larson, et. al., "Three-dimensional x-ray structural microscopy with submicrometre resolution," Nature, 415, (2002), 887-890.

6. W. Ludwig, et. al., "X-ray diffraction contrast tomography: a novel technique for threedimensional grain mapping of polycrystals. I. Direct beam case," Journal of Applied Crystallography, 41(2), (2008), 302-309.

7. A. King, et. al., "Observations of intergranular stress corrosion cracking in a grain-mapped polycrystal," Science, 321(5887), (2008), 382-385.

8. U. Lienert, et al., "High-energy diffraction microscopy at the advanced photon source," JOM, 63(7) (2011), 70-77.

9. R.M. Suter, et. al., "Forward modeling method for microstructure reconstruction using x-ray diffraction microscopy: Single-crystal verification," Review of Scientific Instruments, 77(12) (2006), 123905.

10. C.M. Hefferan, et. al., "Observation of recovery and recrystallization in high-purity aluminum measured with forward modeling analysis of high-energy diffraction microscopy," Acta Materialia, 60(10) (2012), 4311-4318.

11. S.F. Li, et al., "Three-dimensional plastic response in polycrystalline copper via near-field high-energy X-ray diffraction microscopy," Journal of Applied Crystallography, 45(6) (2012), 1098-1108

12. R. Pokharel, et. al., "Polycrystal Plasticity: Comparison Between Grain-Scale Observations of Deformation and Simulations," Annu. Rev. Condens. Matter Phys, 5 (2014), 317-46.

13. J. Lind, et. al. "Tensile twin nucleation events coupled to neighboring slip observed in 3D," Acta Materialia, (2014), in press.

14. S.F. Li and R. M. Suter, "Adaptive reconstruction method for three-dimensional orientation imaging," Journal of Applied Crystallography, 46(2) (2013), 512-524.

15. Frank, F.C., "Orientation Mapping," Metallurgical Transactions A, 19(3) (1988), 403-408.

16. D.C. Larbalestier, et. al., "Strongly linked current flow in polycrystalline forms of the superconductor MgB2," Nature 410(6825) (2001), 186-189.

17. L. Lu, "Ultrahigh strength and high electrical conductivity in copper," Science 304(5669) (2004), 422-426.

18. L.C. Lim and T. Watanabe, "Fracture toughness and brittle-ductile transition controlled by grain boundary character distribution (GBCD) in polycrystals." Acta metallurgica et materialia, 38(12) (1990), 2507-2516.

19. M.A. Arafin, and J. A. Szpunar, "A new understanding of intergranular stress corrosion cracking resistance of pipeline steel through grain boundary character and crystallographic texture studies," Corrosion Science, 51(1) (2009): 119-128. 
20. S. Ranganathan, "On the geometry of coincidence-site lattices," Acta Crystallographica, 21(2) (1966), 197-199.

21. C.M. Hefferan, "Measurement of Annealing Phenomena in High Purity Metals with Nearfield High Energy X-ray Diffraction Microscopy," PhD diss., Carnegie Mellon University, 2012.

22. CGAL, Computational Geometry Algorithms Library. http://www.cgal.org.

23. P. Alliez, et al., "3D mesh generation," CGAL user and reference manual. CGAL editorial board 3, (2011).

24. S.F. Li, "Imaging of Orientation and Geometry in Microstructures: Development and Applications of High Energy X-ray Diffraction Microscopy," PhD diss., Carnegie Mellon University, 2011.

25. S.F. Li, et al., "Quadruple nodes and grain boundary connectivity in three dimensions," Acta Materialia, 64, (2014), 220-230.

26. J.K. Mackenzie, "Second paper on statistics associated with the random disorientation of cubes," Biometrika, 45(1-2), (1958), 229-240.

27. V. Randle, The measurement of grain boundary geometry (Institute of Physics Pub., 1993).

28. D.L. Olmsted, S.M. Foiles, and E.A. Holm, "Survey of computed grain boundary properties in face-centered cubic metals: I. Grain boundary energy," Acta Materialia, 57(13) (2009), 3694-3703.

29. D.L. Olmsted, S.M. Foiles, and E.A. Holm, "Survey of computed grain boundary properties in face-centered cubic metals: II. Grain boundary mobility," Acta Materialia, 57(13) (2009), 3704-3713.

30. B.W. Reed, et al., "The structure of the cubic coincident site lattice rotation group," Acta Crystallographica Section A: Foundations of Crystallography, 60(3) (2004), 263-277.

31. C.M. Hefferan, et al. "Statistics of high purity nickel microstructure from high energy X-ray diffraction microscopy," Computers, Materials, \& Continua, 14(3) (2010), 209-220.

32. D.G. Brandon, "The structure of high angle grain boundaries," Acta Metallurgica, 14 (1966), 1479-1484.

33. C-S Kim, A.D. Rollett, and G.S. Rohrer, "Grain boundary planes: New dimensions in the grain boundary character distribution," Scripta materialia, 54(6) (2006), 1005-1009.

34. A.D. Spear, et. al., "3D microscale characterization and crystal-plastic FE simulation of fatigue-crack nucleation and propagation in an aluminum alloy," Proceedings of $M S \& T$ 2013, accepted.

35. A. Cerrone, et.al., "Implementation and verification of a microstructure-based capability for modeling microcrack nucleation in LSHR at room temperature," 2014, in preparation.

36. C.A. Stein, et.al., "Fatigue crack initiation, slip localization and twin boundaries in a nickelbased superalloy," 2014, in preparation. 